\title{
Estimating bias in the OCO-2 retrieval algorithm caused by 3-D radiation scattering from unresolved boundary layer clouds
}

\author{
A. Merrelli ${ }^{1}$, R. Bennartz ${ }^{1,2}$, C. W. O'Dell ${ }^{3}$, and T. E. Taylor ${ }^{3}$ \\ ${ }^{1}$ Space Science and Engineering Center, University of Wisconsin-Madison, Madison, Wisconsin, USA \\ ${ }^{2}$ Dept. of Earth and Environmental Science, Vanderbilt University, Nashville, Tennessee, USA \\ ${ }^{3}$ Dept. of Atmospheric Science, Colorado State University, Fort Collins, Colorado, USA
}

Correspondence to: A. Merrelli (aronne.merrelli@ssec.wisc.edu)

Received: 24 September 2014 - Published in Atmos. Meas. Tech. Discuss.: 21 November 2014

Revised: 11 March 2015 - Accepted: 11 March 2015 - Published: 1 April 2015

\begin{abstract}
Due to the complexity of the multiple scattering problem for shortwave radiative transfer in Earth's atmosphere, operational physical retrieval algorithms commonly use a plane parallel radiative transfer model (RTM). This so-called one-dimensional (1-D) assumption allows practical retrieval algorithms to be implemented. In order to understand the impacts of this assumption for low altitude, unresolved clouds observed by OCO-2, the three-dimensional (3-D) radiative transfer model SHDOM is used to generate synthetic observations which are then processed by the operational retrieval algorithm based on a 1-D RTM. Simulations are performed over three realistic surface spectral albedos, corresponding to snow, vegetation, and bare soil. The results show that the existing cloud screening algorithm has difficulty identifying sub-field of view (FOV), unresolved clouds that fill less than half of the FOV. The unresolved clouds introduce a bias in the retrieved $\mathrm{CO}_{2}$ concentration, as quantified by the dry air mole fraction $\left(X_{\mathrm{CO}_{2}}\right)$. The biases are relatively small (less than $1 \mathrm{ppm}$ ) when the albedo at $2.1 \mu \mathrm{m}$ is high, which is common over bare land surfaces. For cases with low $2.1 \mu \mathrm{m}$ albedo, such as snow, the bias becomes much larger, up to $5 \mathrm{ppm}$. These results indicate that the $X_{\mathrm{CO}_{2}}$ retrieval appears robust to 3-D scattering effects from unresolved low level clouds when the short wave infrared surface albedo is large, but for darker surfaces these clouds can introduce significant biases.
\end{abstract}

\section{Introduction}

Constraining carbon dioxide fluxes on regional scales has been an important goal of the earth science community for many years. Increased knowledge in this area will improve our current understanding of the different sources and sinks in the global carbon cycle, as well as improve projections in future climate change scenarios. Recently, efforts have been focused on accurate measurements of carbon dioxide concentration from satellite platforms, in order to obtain global data sets to constrain flux inversion models. Measuring carbon dioxide in the shortwave region, by observing reflected solar radiation in near infrared carbon dioxide absorption bands, has the advantage of sensitivity to the full atmospheric column. Relying on the thermal emission bands reduces sensitivity to boundary layer carbon dioxide, where most of the concentration variations reside.

Several satellite sensors have used this technique, including the past sensor SCanning Imaging Absorption spectroMeter of Atmospheric CHartographY (SCIAMACHY) on the European Space Agency's ENVIronmental SATellite (ENVISAT) (Buchwitz et al., 2007), the current Thermal and Near Infrared Sensor for Carbon Observation FourierTransform Spectrometer (TANSO-FTS) on the Greenhouse gases Observing SATellite (GOSAT) (Yoshida et al., 2011), and the Orbiting Carbon Observatory-2 (OCO-2) (Crisp et al., 2004; Crisp and Johnson, 2005), and the planned mission CarbonSat (Bovensmann et al., 2010). The current state of the art retrieval technique uses a combined observation of the oxygen A-band at $0.76 \mu \mathrm{m}$, and $\mathrm{CO}_{2}$ absorption bands 
at 1.6 and $2.1 \mu \mathrm{m}$, in order to estimate the column-averaged dry air mole fraction of $\mathrm{CO}_{2}$, termed $X_{\mathrm{CO}_{2}}$. This definition of $X_{\mathrm{CO}_{2}}$ is consistent with the OCO-2 retrieval algorithms (O'Dell et al., 2012) and the Total Carbon Column Observing Network (TCCON, Wunch et al., 2011a). Each absorption band is observed at high spectral resolution $\left(\mathrm{O}\left(10^{3}\right)\right.$ points per band), to increase the measurement's information content.

In clear air columns, this technique is relatively straightforward, and can be used for various trace gas concentration retrievals by observing their associated molecular absorption bands. For carbon dioxide, the measurement is especially challenging, since the desired measurement uncertainty is very small. Miller et al. (2007) estimates an absolute accuracy of 1-2 ppm is required for $X_{\mathrm{CO}_{2}}$ (a relative accuracy of approximately $0.25-0.5 \%$ ) in order to substantially reduce surface flux uncertainties. Any retrievals must account for variations in other geophysical variables that can alter the signal (aerosols, surface albedo, and clouds, for example), or detect their presence in order to appropriately flag the data as contaminated.

The retrieval problem is an especially challenging one due to a combination of high complexity of the radiative transfer problem and high dimensional spectral data. The radiative transfer model must deal with multiple scattering, and for OCO-2, polarization. The high spectral resolution measurement covers regions with many isolated absorption lines where the individual absorption line shapes and the instrument spectral response become extremely important. All practical retrieval methods make the plane parallel assumption to simplify the radiative transfer problem to one dimension (1-D). By making this assumption, the atmosphere is assumed to be horizontally homogeneous. The impact of this 1-D assumption has not been well explored in the context of trace gas retrievals, although it has been studied in other contexts, such as retrieval of aerosol near cloud (Várnai et al., 2013) or cloud properties in inhomogeneous cloud fields (Zhang et al., 2012).

In this study, we investigate how the 1-D radiative transfer assumption affects $X_{\mathrm{CO}_{2}}$ retrieval in a specific case: observations of isolated low clouds with simulated OCO-2 observations. By using a three-dimensional (3-D) radiative transfer model, we generate realistic spectra for this particular case where the sensor's view contains strong 3-D effects such as cloud shadowing and lateral photon transport.

The paper is organized as follows: in Sect. 2 the radiative transfer models and input data sets are described in detail. This includes a validation analysis to show the new simulation framework is equivalent to an existing OCO-2 simulation program. Important characteristics of the OCO-2 instrument are reviewed. In Sect. 3, the operational OCO-2 retrieval algorithm is reviewed, with emphasis on the cloud screening methods. Section 4 describes the specific simulated scenarios, and the results of processing the synthetic spectra with the retrieval algorithm are presented in Sect. 5. Finally, the results are discussed and summarized in Sect. 6.

\section{Simulating OCO-2 observations}

\subsection{OCO-2 sensor characteristics}

The OCO-2 sensor measures radiance spectra in three narrow spectral bands: the oxygen A-band in the near infrared (NIR) at $0.76 \mu \mathrm{m}$ wavelength, and the $\mathrm{CO}_{2}$ bands in the short wave infrared (SWIR) at 1.6 and $2.1 \mu \mathrm{m}$. These are referred to as the $\mathrm{O} 2 \mathrm{~A}, \mathrm{WCO} 2$ ("weak" $\mathrm{CO}_{2}$ ) and $\mathrm{SCO} 2$ ("strong" $\mathrm{CO}_{2}$ ) bands, for brevity. Each of the three bands is a 1016 point spectrum covering the absorption feature. This produces spectral channel spacings of approximately 0.015 , 0.03 , and $0.04 \mathrm{~nm}$ in the middle of the three bands. The instrument contains a linearly polarized filter that will be oriented perpendicularly to the principal plane throughout the orbit, so that the sensor measures the combination of Stokes vector components $(I-Q) / 2$. The sensor will be operated in three modes: "nadir", "glint", and "target". In nadir mode, the sensor will point toward the subsatellite point (rotating to keep the polarizer orientation as described above $)^{1}$. This is the primary mode for over-land retrievals. In glint mode, the sensor will point near the sun glint point, with the exact angular separation from the glint point to be determined during on-orbit tests. This is the primary mode for over-ocean retrievals, as the ocean surface is very dark in the SWIR spectral range, only reflecting sufficient solar radiation near the glint point. In target mode, the sensor will repoint through the orbit to keep a particular point on Earth's surface in view. Note that the instrument pointing is achieved through precise control of the satellite bus yaw, pitch and roll axes via onboard reaction wheels; i.e., there are no moving fore-optics components. This study focuses on the nadir mode, so the glint and target mode are not discussed further.

Lab measurements of the OCO-2 instrument line shape (ILS) are used to compute the simulated sensor radiance from monochromatic calculations. The measured ILS shapes have full width at half maximum (FWHM) of 0.04, 0.08, and $0.1 \mathrm{~nm}$, in the three bands, implying a spectral resolution $(R \sim \lambda / \Delta \lambda)$ of 20000 . No measurements of the spatial response function (SRF) were available for use in this study, so an approximation was used as a surrogate for the real SRF. The surrogate SRF first combines the rectangular slit field of view with the spacecraft motion through the $1 / 3 \mathrm{~s}$ collection time. When the slit is oriented perpendicularly with respect

\footnotetext{
${ }^{1}$ Due to a design error, the instrument's polarization response is rotated 90 degrees with respect to the original specification. The instrument will orbit in the originally specified orientation for nadir mode, implying the polarized measurement is $(I+Q) / 2$. In glint node, the spacecraft will be rotated 60 degrees from the originally specified orientation, yielding a polarized measurement of $(I+1 / 2 Q-\sqrt{3} / 2 U) / 2$.
} 
to the spacecraft motion, the resulting convolution of the slit field of view (FOV) and spacecraft motion is a rectangle of about $1.3 \mathrm{~km} \times 2.3 \mathrm{~km}$ on the Earth surface. For other orientations, the convolution will yield a parallelogram shape. Since the slit is oriented according to the scattering principal plane, the relative orientation changes through the orbit. For simplicity, the perpendicular slit orientation, and rectangular IFOV (Instantaneous Field Of View), is used for all simulations. This rectangular SRF is then convolved with a circular 2-D Gaussian function with $\sigma$ width of $0.6 \mathrm{~km}$ for the nadir view, to approximate the optical blur of the sensor. The result is an oval shaped SRF, with widths at half-maximum of $1.7 \mathrm{~km} \times 2.3 \mathrm{~km}$. This surrogate $\mathrm{SRF}$ ignores some additional detailed information about the shape of the SRF due to focal plane readout timing, but these further details are likely not important for this study, as long as the SRF size and profiles are approximately accurate.

\subsection{Radiative transfer models}

During algorithm development support for the original OCO mission, an OCO simulator was developed at Colorado State University to produce full orbit simulations (O'Brien et al., 2009). Development on the simulator has continued through the NASA Atmospheric $\mathrm{CO}_{2}$ Observations from Space (ACOS) program, and it has been updated to support OCO-2. The simulator uses a highly optimized plane-parallel radiative transfer model to compute radiance in scattering atmospheric profiles, including cloud and aerosol layers. The overall simulator processing pipeline was used as a template while implementing the 3-D version used in this study. In short, the 3-D version replicates the same steps as the 1-D model but replaces the 1-D plane parallel radiative transfer model with the spherical harmonic discrete ordinate method (SHDOM) developed by Evans (1998). For brevity, the two methods will be called the 1-D OCO-2 and 3-D OCO-2 simulators.

\subsection{Inputs to 3-D OCO-2 simulator}

Important advances in spectroscopy have been made to effectively model the OCO-2 bands. The OCO-2 science team supports development of advanced absorption line models including broadening and line mixing effects (Tran and Hartmann, 2008; Thompson et al., 2012). The gas absorption models are used to produce look up tables across the OCO2 bands at a frequency resolution of $0.01 \mathrm{~cm}^{-1}$ wave number, for a representative range of atmospheric pressures and temperatures. The frequency dimension is thinned with an optimal subset that uses coarse spacing (up to $2 \mathrm{~cm}^{-1}$ ) in the outer line wings, and the full $0.01 \mathrm{~cm}^{-1}$ spacing at line centers. This optimal subset defines the monochromatic frequency set for individual radiative transfer model runs.

At these monochromatic frequencies, the scattering properties are computed from a Mie algorithm (using the support programs supplied with SHDOM) for cloud liquid water and dust aerosol. For cloud liquid water, a gamma distribution particle size distribution (PSD) is used:

$n(r)=a r^{\alpha} \exp (-b r)$.

The $\alpha$ parameter is set to 7 , which is equivalent to an effective variance of 0.1 (Hansen and Travis, 1974). The effective radius is set to $10 \mu \mathrm{m}$. The aerosol is defined by the dust type within the 1-D OCO-2 simulator, which uses the dust aerosol data from Dubovik et al. (2002) from Solar Village, Saudi Arabia. This is a scattering aerosol $(\omega \sim 0.96)$, with effective radii of 0.1 and $1.9 \mu \mathrm{m}$ in the fine and coarse modes, respectively.

SHDOM is run at each of the monochromatic frequencies in the optimal sampling subset, and computes normalized reflectance. Since the optimal sampling list is a sparse subset of the $0.01 \mathrm{~cm}^{-1}$ monochromatic grid, the SHDOM simulations are linearly interpolated to get continuous sampling. These reflectances are multiplied by the solar irradiance and then convolved with the per-channel measured ILS, where the irradiance and ILS are both defined on the continuous $0.01 \mathrm{~cm}^{-1}$ grid. The result is a spectral data array with 3 dimensions ( $X, Y$, and spectral frequency), where the $X$ and $Y$ grid is defined by the $100 \mathrm{~m}$ 3-D grid defining the liquid water content (LWC) and aerosol field for SHDOM. One such spectral data array is simulated for each of the OCO-2 bands.

\subsection{Validation with 1-D OCO-2 simulator}

In order to validate the radiance spectra produced by the 3-D OCO-2 simulator, a simple test was performed where the input grid to both simulators was an identical clear atmosphere column. In this case the SHDOM will essentially reproduce a 1-D radiative transfer calculation. The differences between the spectra from each simulator were very small. Figure 1 shows an example OCO-2 observation, with the difference spectra between the OCO-2 simulator and the 3-D simulation from SHDOM. Note the $y$ axis range on the difference plot is 3 orders of magnitude smaller than the $y$ axis range on the radiance spectra, and the red lines show the $\pm 1 \sigma$ lines from the sensor noise model.

\section{ACOS retrieval algorithm}

The $X_{\mathrm{CO}_{2}}$ retrieval for OCO-2 was developed by the NASA ACOS team. The algorithm uses an optimal estimation physical retrieval, with screening algorithms applied to identify the subset of the full observation set that will produce high quality $X_{\mathrm{CO}_{2}}$ retrievals. It has been applied to GOSAT NIR and SWIR spectral measurements, which have similar spectral characteristics to the OCO-2 measurements. 

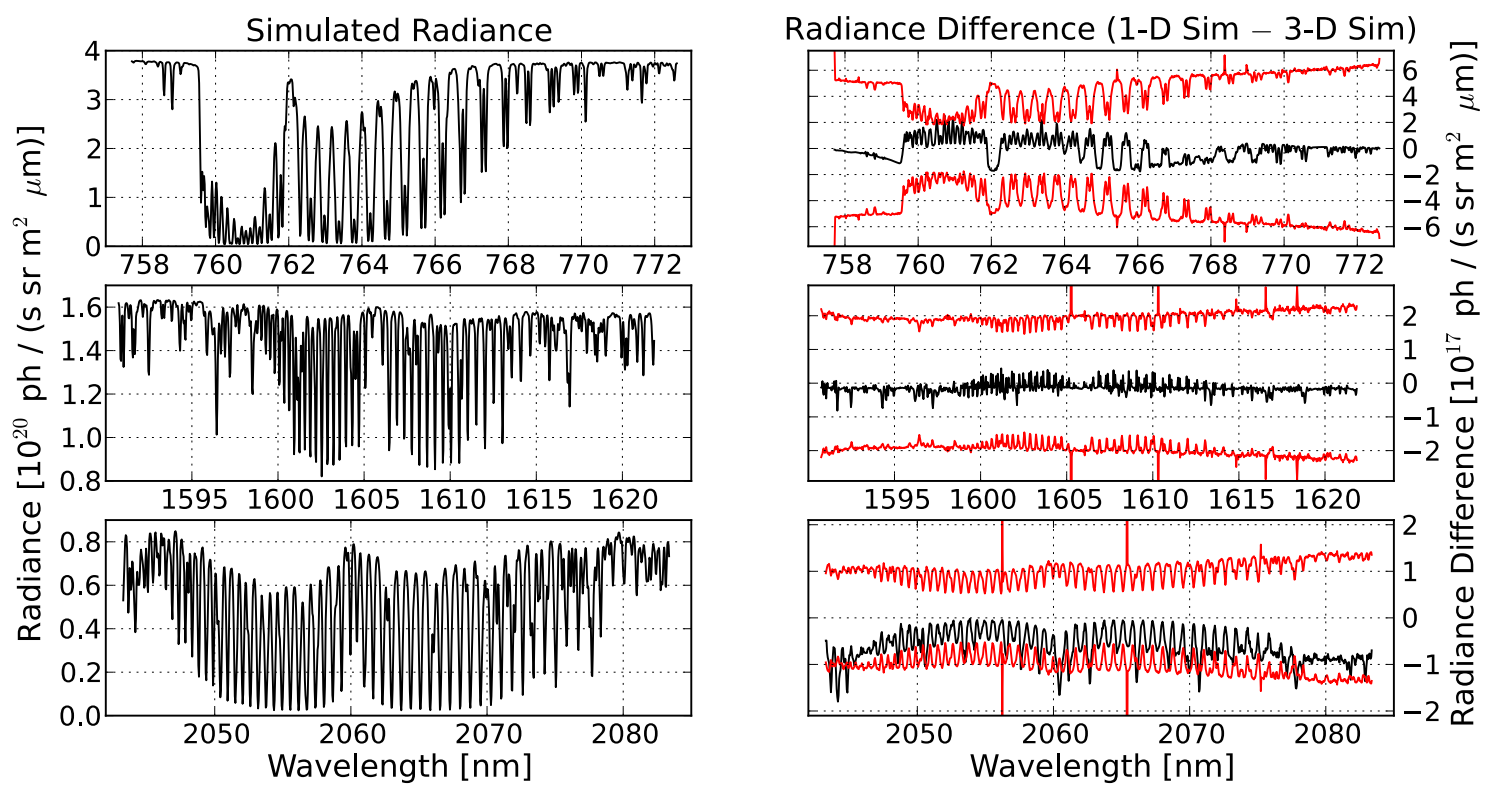

Figure 1. Comparing simulated radiance spectra for a clear atmosphere column using the 1-D OCO-2 simulator and the 3-D SHDOM-based OCO-2 simulator. The left column shows the simulated radiance for each band, and the right column shows the radiance differences (1-D minus 3-D simulated spectra), with the $\pm 1 \sigma$ sensor noise level envelope overplotted with red lines. Note the $y$ axis units for the plots in the left column are a factor of $10^{3}$ larger than the units on the right column.

\subsection{Physical optimal estimation retrieval}

The overall design of the optimal estimation (OE) retrieval is described in O'Dell et al. (2012), Crisp et al. (2012) and Crisp et al. (2010). For this study, the B3.4 version of the algorithm is used, which has significant differences compared to the B2.9 algorithm documented in O'Dell et al. (2012). The newer algorithm retrieves two state variables related to fluorescence and uses a different aerosol parameterization. No fluorescence was included in the 3-D OCO2 simulator, so this part of the retrieval is disabled and the fluorescence variable values within the prior and first guess state vector are set to zero. In both the B2.9 and B3.4 versions, four atmospheric scatterers are used: water cloud, ice cloud, and "Kahn $2 b$ " and "Kahn $3 b$ " aerosols. The Kahn aerosol types are defined in Kahn et al. (2001). In the B3.4 version, the scattering particles use a parameterized profile shape instead of allowing the profile to vary independently across pressure levels. For each of the four scatterer types, the profile is parameterized with a vertical Gaussian profile in optical depth, with three free parameters: the amplitude (the total optical depth), thickness, and height. The thickness and height are expressed in terms of pressure normalized by the surface pressure. The Gaussian profile is truncated when the vertical offset is near or below the surface, in which case the profile is renormalized such that the integral is equal to the total optical depth. Figure 2 shows the prior profiles used in the retrieval along with the $1-\sigma$ perturbations to the total optical depth and height from the a priori covariance. The assumed prior values and variances are listed in Table 1. All covariances are assumed
Table 1. A priori values and SDs for aerosol and cloud profiles. Note that the state vector contains the logarithm of the optical depth, so the $\sigma$ range is a multiplicative scaling. The pressure heights and thicknesses are expressed as unitless ratios with the surface pressure.

\begin{tabular}{llll}
\hline $\begin{array}{l}\text { State } \\
\text { Variable }\end{array}$ & Particle & $\begin{array}{l}\text { A Priori } \\
\text { Value }\end{array}$ & $\begin{array}{l}\text { A Priori } \\
1-\sigma \text { error }\end{array}$ \\
\hline \multirow{3}{*}{ Optical Depth } & Cloud Water & 0.0125 & 6.0 \\
& Cloud Ice & 0.0125 & 6.0 \\
& Kahn 2b, 3b & 0.0125 & 6.0 \\
\hline \multirow{3}{*}{ Pressure Height } & Cloud Water & 0.75 & 0.4 \\
& Cloud Ice & 0.3 & 0.2 \\
& Kahn 2b, 3b & 1.0 & 0.4 \\
\hline \multirow{3}{*}{ Pressure Thickness } & Cloud Water & 0.1 & 0.01 \\
& Cloud Ice & 0.04 & 0.01 \\
& Kahn 2b, 3b & 0.2 & 0.01 \\
\hline
\end{tabular}

to be zero. Note that the thickness is tightly constrained by the prior variance and is effectively a fixed parameter. This parameterization results in a maximum number of degrees of freedom of 8 for the scattering particles.

\subsection{Pre-screening algorithm}

The radiative transfer algorithm at the heart of the $\mathrm{OE}$ retrieval is a plane parallel code similar to the 1-D OCO2 simulator, but further simplified and optimized. On a 


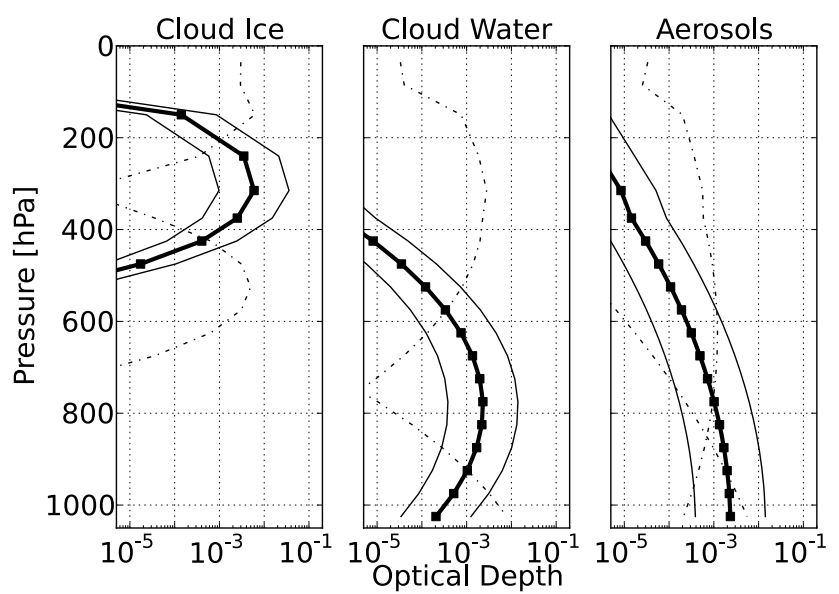

Figure 2. Prior optical depth profiles. The thick solid line with square markers shows the per-layer optical depth prior profile; the solid lines show the $\pm \sigma$ range applied to the optical depths; the dotdashed lines show the $\pm \sigma$ range applied to the pressure heights.

standard workstation at the University of Wisconsin (AMD Opteron $^{\mathrm{TM}} 4180 \mathrm{CPU}$ ), this simplified model takes on the order of $30 \mathrm{~s}$ to compute one iteration of the $\mathrm{OE}$ algorithm (e.g., computing the forward model result and Jacobians at the state vector update). Compared to the data collection rate (24 observations per second), this implies that only a small fraction of the observations will be processed with the physical OE retrieval. The target for initial operating capability is to apply the physical retrieval to $6 \%$ of the total observations. Thus, careful prescreening methods must be applied to the observations to select the subset most likely to produce accurate $X_{\mathrm{CO}_{2}}$ retrievals. The precise form of the screening method is itself a subject of research within the ACOS effort (Mandrake et al., 2013). In this study, the focus will be on the "A-band preprocessor" (ABP), described in Taylor et al. (2012). This prescreening algorithm uses a highly simplified, single-step retrieval of surface pressure and surface albedo, assuming a clear sky profile with only Rayleigh scattering. It uses a subset of the full A-band spectrum. Tests with this algorithm showed that the difference between the retrieved surface pressure and the interpolated ECMWF analysis surface pressure is an effective cloud screen. The pressure difference is especially sensitive to higher altitude clouds that introduce large photon path modification. Observations that yield retrieved surface pressures much different than the forecast value $(> \pm 10 \mathrm{hPa})$ indicate significant cloud and/or aerosol contamination in the observed radiance (O'Dell et al., 2012). In effect, a thick cloud layer can act as a surface, so that the retrieved surface pressure is related to the cloud top pressure (CTP). This approach has been proposed as a retrieved cloud product for previous missions (for example, a CTP measurement for the $\mathrm{O}_{2}$ A-band was originally planned for CloudSat, see Miller and Stephens, 2001). For OCO-2, however, the retrieved surface pressure will be primarily used to re- move cloud-contaminated observations via the ABP. At this time is not possible to retrieve $X_{\mathrm{CO}_{2}}$ to the required accuracy from the cloud-contaminated observations.

Following Taylor et al. (2012) and simulation tests with the B3.4 algorithm, a surface pressure threshold of $10 \mathrm{hPa}$ is used for the ABP. In addition, a $\chi^{2}$ threshold is computed by the ABP, based on the noise level predicted by the sensor noise model (O'Dell et al., 2011) and the radiance level of the observation. If the computed $\chi^{2}$ is larger than the threshold, the observation will be screened.

Cloud and aerosol contamination need not be directly in the sensor's field of view, and may be produced by significant multiple scattered radiation from clouds near the field of view. Such scattered radiation could cause either threshold (surface pressure or $\chi^{2}$ ) to be exceeded. In this more general sense, the ABP could be viewed as detecting "photon path modification" in the radiance observation, and thus the 1-D retrieval algorithm will not produce an accurate $X_{\mathrm{CO}_{2}}$ retrieval.

\subsection{Post-screening algorithm}

In cases where the prescreening method does not correctly detect a cloud or aerosol contaminated spectrum, the retrieval may converge to an inaccurate $X_{\mathrm{CO}_{2}}$ result. Therefore, additional post-screening is performed on the retrieved state vector in an attempt to eliminate poor quality retrievals. These post screens are generally made by performing threshold tests on the retrieved surface pressure, surface albedos, retrieved aerosol or cloud optical depths, and reduced $\chi^{2}$ values for each band. The reduced $\chi^{2}$ is the calculated $\chi^{2}$ value divided by the number of degrees of freedom, which is expected to be close to 1 if the modeled and measured spectra differ only by random sensor noise. The first two post screen threshold tests are very similar to those used by the ABP, but use tighter threshold values. The latter two thresholds are motivated from simulation tests (O'Dell et al., 2012).

The values of the various post screening thresholds are continually adjusted for the ACOS products by analysis and comparisons of GOSAT and TCCON retrievals, in order to minimize the bias in the final retrieved $X_{\mathrm{CO}_{2}}$. Thus the particular values prescribed in the B3.4 release documentation are not necessarily relevant to these simulation experiments, especially for the reduced $\chi^{2}$ thresholds for the spectral residuals. The residuals will include various model deficiencies (such as inaccurate absorption line spectroscopy) when compared with real data. No such effects are included in this simulation and retrieval framework, so the reduced $\chi^{2}$ values will be close to the theoretical values. To derive $\chi^{2}$ thresholds for these tests, an ensemble of simulated clear sky spectra (with aerosols but no cloud liquid water) was processed with the L2 algorithm. A threshold of 1.25 was selected for all three bands, which is 3.6 SDs above the mean reduced $\chi^{2}$ values over all retrievals. Approximately $0.1 \%$ of all the clear retrievals exceed this threshold in one of the bands. 
Screening thresholds on the retrieved scattering optical depths are also important for high accuracy retrievals. The values used in B3.4 applied to GOSAT high-gain observations over land are 0.15 for the sum of all four scatterers (cloud water, cloud ice, and both Kahn aerosol types), 0.07 for cloud water, and 0.045 for cloud ice. For the GOSAT medium-gain observations over land, these thresholds are $0.1,0.07$, and 0.03 , for the total, cloud water, and cloud ice optical depths, respectively. Since these thresholds were empirically determined for the GOSAT data as processed with the B3.4 algorithm, they are not necessarily the best choice for this analysis. Tests with the clear sky retrievals show that these thresholds were somewhat too restrictive, and the selected thresholds in this analysis are $0.175,0.1$, and 0.03 for the total, cloud water, and cloud ice optical depths, respectively. These threshold values are consistent with the values selected for simulation-based tests (O'Dell et al., 2012), and are still more restrictive than the initially reported threshold of 0.3 in aerosol optical depth for performing $X_{\mathrm{CO}_{2}}$ retrievals (Crisp et al., 2004).

\section{Retrieval test setup}

The specific tests are designed to explore potential retrieval biases caused by low level clouds. Even without considering 3-D scattering effects, low level layer clouds can be challenging to identify (O'Dell et al., 2012). A low level layer cloud can introduce both photon path shortening and lengthening (Bennartz and Preusker, 2006), which can tend to cancel and mask the cloud's effect on the radiance measurement. The work presented here can be viewed as an extension of these previous 1-D simulation efforts to broken or isolated low clouds, which have strong 3-D scattering effects.

\subsection{Scenarios}

A single spheroidal liquid water cloud was created in the center of a $3.5 \mathrm{~km}$ (horizontal) $\times 2.5 \mathrm{~km}$ (vertical) domain. The grid spacing is $100 \mathrm{~m}$ in all three dimensions. Dust aerosol is evenly distributed within the domain, with a total aerosol optical depth (AOD) of 0.05 in the vertical dimension. The cloud has an altitude of $1.6 \mathrm{~km}$, and a fixed thickness of 0.6 $\mathrm{km}$ in the $z$ axis. Three cloud sizes are simulated, with the cloud diameter in the horizontal axes set to $0.6,0.8$ and 1.2 $\mathrm{km}$. Therefore the smallest cloud is spherical, while the 0.8 and $1.2 \mathrm{~km}$ clouds are oblate spheroids. The liquid water content (LWC) has a quadratic profile in all three dimensions. The cloud fraction within the blurred SRF (see Sect. 2.1) is not well defined, but it covers roughly 10,20 , and $40 \%$ of the unblurred, rectangular nadir FOV for the three cloud sizes. The maximum LWC, in the center of the cloud, is $0.125 \mathrm{~g} \mathrm{~m}^{-3}$, yielding a maximum liquid water path (LWP) through the cloud center of $50 \mathrm{~g} \mathrm{~m}^{-2}$. The maximum visible optical depth along this same path is approximately 8 . Two
Table 2. Lambertian surface albedos, in each of the three OCO-2 spectral bands, for the three surface types.

\begin{tabular}{lccc}
\hline Surface type & $\mathrm{O}_{2}$ A-band & Weak $\mathrm{CO}_{2}$ & Strong $\mathrm{CO}_{2}$ \\
\hline Snow & 0.87 & 0.10 & 0.02 \\
Vegetation & 0.34 & 0.22 & 0.10 \\
Soil & 0.25 & 0.35 & 0.31 \\
\hline
\end{tabular}

solar zenith angles (SZAs) are used: 35 , and $60^{\circ}$. The surface is assumed to be Lambertian, with realistic albedo values in regions classified as snow, vegetated, and soil. Table 2 lists the selected values.

The SHDOM has the option to run with open or periodic boundary conditions. The periodic boundary condition is used for all cases. SHDOM is used to simulated a nadir view using these LWC and aerosol fields. The cloud field is then a grid of identical clouds with a $3.5 \mathrm{~km}$ horizontal spacing, rather than a single isolated cloud. In Fig. 3, the nadir sensor view is shown for the three selected surface albedos for a single OCO-2 spectral channel in each of the three bands. The single channel is chosen at a wavelength between absorption lines so there is little extinction of the solar radiation. The views are shown for the $35^{\circ}$ solar zenith angle.

\subsection{Creating OCO-2 spectra}

Using the spectral data array described in Sect. 2.3, a synthetic OCO-2 observation is generated by applying the surrogate SRF (see Sect. 2.1). The 2-D nadir radiance array at each channel frequency is multiplied by the SRF and summed to produce the OCO-2 channel radiance. Figure 4 shows examples of the SRF applied to a low absorption O2A band channel. The SRF is applied at various positions, by scanning left-to-right over the cloud in the scene. A total of 25 SRF centered positions are used, where the SRF is shifted by a single $100 \mathrm{~m}$ grid cell, and the 10th position is centered in the $3.5 \mathrm{~km}$ domain. Positions $0,5,10,15$ and 20 (i.e., the center of the SRF falls at positions $0.75,1.25,1.75,2.25$ and $2.75 \mathrm{~km}$ along the $x$ axis in the $100 \mathrm{~m}$ grid) are shown in Fig. 4 . The SRF positions move between regions dominated by clear, cloudy or shadowed high resolution grid cells, depending on the cloud size and solar zenith angle. For brevity, the positions will be referred to as clear, cloudy and shadow, depending on which type of high resolution pixel is dominant at that location, but it is apparent that in all cases the SRF will contain a mixture of all three types.

The process described in the previous section yields a total of 25 spectra, one per SRF positions for each scenario (a selected solar zenith angle and surface albedo). For each spectrum, an ensemble of 37 simulated OCO-2 observations is generated with independent sensor noise realizations for each observation. The sensor noise is drawn from a Gaussian Probability Distribution Function, scaled by the sensor noise model from O'Dell et al. (2011). Each of these ensembles 


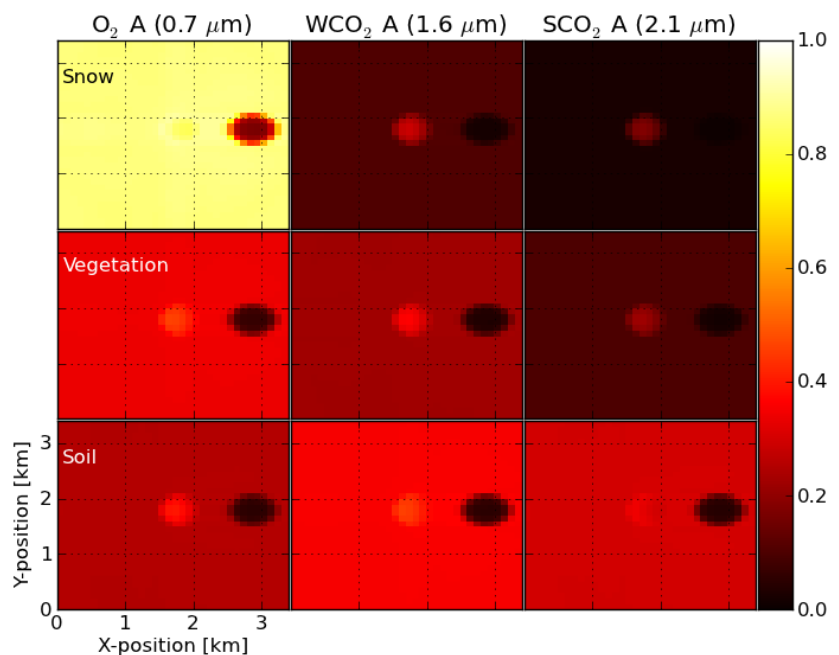

Figure 3. Overhead views of the scene reflectance as simulated by SHDOM, for a channel within each OCO-2 band that has relatively low gas absorption. Each of the three columns shows the reflectance for one of the three OCO-2 bands, and each of the three rows shows reflectance from one surface type. All images are shown with the same display scale, to highlight the differences in contrast between the cloud and surface for the different spectral bands and surface types.

are processed with the ABP algorithm and the B3.4 L2 retrieval code, and the relevant quantities are averaged over the ensemble members. The ABP uses a subset of the O2A band $(759.18-760.75 \mathrm{~nm})$, while the $\mathrm{L} 2$ retrieval runs on the full 3-band spectra. The L2 retrieval uses an input meteorology data set (in operations, this is taken from a numerical weather forecast), which is set to the truth values. In other words, the meteorology sent to the L2 retrieval is the same meteorology data used to simulate the spectra with the 3-D OCO-2 simulator.

\section{Retrieval test results}

\subsection{Screening rates}

In normal operations, the results of the ABP would be applied before performing L2 retrievals. In cases where 3-D effects are important, it is possible that the ABP could preferentially identify cloudy observations in cases where the full L2 algorithm can produce accurate retrievals. This behavior would not have been captured in the development of the ABP, since all radiative transfer models used in the earlier studies were plane parallel. Therefore, in these experiments, the L2 algorithm is applied to all simulated observations, regardless of the output from the ABP. This allows full characterization of the ABP performance, to verify that it is removing the cloud-contaminated observations that the L2 algorithm cannot accurately process.

\subsubsection{ABP prescreening}

Recall that at each SRF position, an ensemble of 37 spectra were generated with independent sensor noise. The ensemble allows for a screening fraction to be computed at each position. Figure 5 shows these fractions as a function of SRF position, for the various scenarios. The ABP's performance appears directly correlated to the $\mathrm{O} 2 \mathrm{~A}$ band albedo and the cloud size, as expected. Recall that the ABP retrieves just the surface pressure from the $\mathrm{O} 2 \mathrm{~A}$ band (see Sect. 3). Over snow, since the surface is much more reflective, a larger proportion of the observed radiance is due to the single scattering off the surface, which will tend to pull the retrieved pressure to the surface value. The ABP also detects a cloud mainly when the SRF is centered on the cloud, but not in the primarily "clear" or "shadow" positions. A single screening rate for each scenario can be computed by averaging over the SRF positions. Table 3 shows these results.

\subsubsection{Postscreener}

Figure 6 shows the postscreening fractions, with the same layout as Fig. 5 showing the prescreening fractions. The postscreening fractions are defined with respect to the total number of simulated observations, since the L2 retrieval was run on all observations, not just the ones that were not identified as cloudy by the ABP. Ideally, the postscreened fractions should be equal or higher than the prescreened fractions from the ABP. This would imply that the ABP correctly identified an observation that would not have produced an accurate L2 retrieval. A higher postscreening fraction is also expected, since the L2 retrieval has access to more information (since it retrieves from all 3 OCO-2 bands), and uses a more complicated state vector.

For the vegetation and soil surfaces with $60^{\circ}$ solar zenith angle, the postscreening fractions as a function of the SRF position are extremely similar to the prescreening fractions. For the remaining cases, except for the soil surface at $35^{\circ}$ solar zenith angle, the post screening method tends to identify a much larger fraction of cloudy observations. This is especially true over snow where the fraction reaches nearly $100 \%$ for the $0.8 \mathrm{~km}$ cloud. The outlier scenario here is the soil surface at $35^{\circ}$ solar zenith angle, where the ABP identifies a much higher rate of cloud contamination than the postscreener. This behavior could be described in two ways: either the ABP could be improperly identifying these observations, (if these retrievals produce accurate $X_{\mathrm{CO}_{2}}$ retrievals) or the postscreener could be failing (if the retrieved $X_{\mathrm{CO}_{2}}$ is not accurate). This behavior will be discussed below in the context of the state variable biases.

A summary of the postscreen fractions is shown in Table 4. Note that although the postscreen identifies the highest fraction of the cloud contaminated observations, it also screens almost $30 \%$ of the clear column test spectra. This suggests 
Table 3. Percentage of simulated observations identified as cloudy by prescreener algorithm.

\begin{tabular}{llcc}
\hline $\begin{array}{l}\text { Surface } \\
\text { Type }\end{array}$ & $\begin{array}{l}\text { Cloud } \\
\text { Size }\end{array}$ & $\begin{array}{c}\text { Prescreen Pct. } \\
\text { SZA }=35^{\circ}\end{array}$ & $\begin{array}{c}\text { Prescreen Pct. } \\
\text { SZA }=60^{\circ}\end{array}$ \\
\hline Snow & clear & 0.0 & 0.0 \\
Snow & $0.6 \mathrm{~km}$ & 0.0 & 0.0 \\
Snow & $0.8 \mathrm{~km}$ & 0.0 & 0.0 \\
Snow & $1.2 \mathrm{~km}$ & & 0.0 \\
\hline Vege & clear & 0.0 & 0.0 \\
Vege & $0.6 \mathrm{~km}$ & 0.0 & 0.0 \\
Vege & $0.8 \mathrm{~km}$ & 0.0 & 0.0 \\
Vege & $1.2 \mathrm{~km}$ & & 40.2 \\
\hline Soil & clear & 0.0 & 0.0 \\
Soil & $0.6 \mathrm{~km}$ & 0.0 & 0.0 \\
Soil & $0.8 \mathrm{~km}$ & 34.6 & 11.6 \\
Soil & $1.2 \mathrm{~km}$ & & 70.3 \\
\hline
\end{tabular}

that the postscreen could be improved with scene dependent thresholds.

\subsection{State variable bias}

In order to quantify the impact of 3-D radiative transfer effects on the retrieved state variables, the retrieval outputs will be compared both to the known truth values as well as the results from the cloud-free simulations. Since the ACOS $X_{\mathrm{CO}_{2}}$ retrieval is known to have biases even for clear sky retrievals (O'Dell et al., 2012; Wunch et al., 2011b), the cloud-free simulations help to separate the bias caused by 3-D radiative transfer effects from the clear sky bias already present in the retrieval. In any particular scenario, the bias due to 3-D effects will be the difference between the bias computed in the cloudy retrievals and the bias computed in the clear sky retrievals.

As an initial example, the previous case noted in the screening discussion above is revisited. For the $0.8 \mathrm{~km}$ cloud, over the soil surface with solar zenith of $35^{\circ}$, the ABP identified all spectra with the SRF centered above the cloud as cloud contaminated. However, the postscreen only found about $20 \%$ of these observations to be cloud contaminated. Figure 7 shows the bias in the retrieved $X_{\mathrm{CO}_{2}}$ as a function of the SRF position for this scenario. The bias is defined as the retrieved values, minus the known truth $X_{\mathrm{CO}_{2}}$ value. The left subplot shows all converged retrievals; the middle plot shows the screened retrievals (both prescreen and postscreen are applied). The set of retrievals shown in the middle plot would be the actual set retrieved by the full end-to-end L2 processing algorithm. The gap in observations around SRF position 10 is due to the ABP. The solid horizontal line shows the mean retrieved $X_{\mathrm{CO}_{2}}$ from the clear sky retrievals performed for this scenario; the bold error bar marker centered on zero bias shows the $1-\sigma$ range predicted by the posterior covariance from the optimal estimation algorithm. Finally, to summarize the scatter plots, the distribution of points is condensed down to a single box plot in the right subplot, and the number of points in each is marked along the bottom axis. The remaining bias distribution plots will match this layout, with the results condensed into the pair of box plots. The $X_{\mathrm{CO}_{2}}$ biases shown here indicate that the $\mathrm{L} 2$ retrieval is able to compute an accurate value $X_{\mathrm{CO}_{2}}$ for all cases in this scenario. This suggests that the ABP may be too aggressive in identifying cloud contamination in this scenario.

\subsection{1 $\quad X_{\mathrm{CO}_{2}}$}

Figure 8 contains the $X_{\mathrm{CO}_{2}}$ retrieval bias summary for all scenarios including the 0.6 and $0.8 \mathrm{~km}$ clouds. The $1.2 \mathrm{~km}$ cloud scenarios are not included here, since the screening rates are high for these cases. The different surface albedos are organized by row, with the snow, vegetated and soil surfaces in the top, middle, and bottom rows, respectively.

For the snow surface, the $X_{\mathrm{CO}_{2}}$ biases are large, especially for the $60^{\circ}$ solar zenith angle. The $0.6 \mathrm{~km}$ cloud case shows a consistent $-5 \mathrm{ppm}$ bias, which is $4 \mathrm{ppm}$ lower than the clear sky bias. In this case only $12.5 \%$ of the observations would be screened. For the $0.8 \mathrm{~km}$ cloud case, the screening does reduce the bias magnitude, from -9 to $-6 \mathrm{ppm}$. This is the only case where the screening algorithms substantially reduce the bias magnitude. Over the vegetated surface, the absolute biases are much smaller. The mean biases are within $2 \mathrm{ppm}$ of truth, and within $3 \mathrm{ppm}$ of the clear sky value. Finally, over soil, the biases are well represented by the clear sky values. All biases are within approximately $0.3 \mathrm{ppm}$ of the clear sky bias.

Overall, the magnitude of the biases seem to be most strongly related to the $\mathrm{SCO} 2$ surface albedo. In practice, an additional postscreen filter on the retrieved $\mathrm{SCO} 2$ is used, which was not used in these tests. A simple cutoff of about 0.05 albedo in the $\mathrm{SCO} 2$ band would essentially remove all the snow observations. The results here suggest the retrievals over snow are the most sensitive to 3-D radiative transfer effects, further supporting the use of a screening threshold in the $\mathrm{SCO} 2$ band.

\subsubsection{Surface albedo}

A key state variable in the optimal estimation retrieval is the surface albedo in each band. The retrieved values are strongly influenced by the 3-D radiative transfer effects. In Fig. 9 the biases for the retrieved $\mathrm{SCO} 2$ albedo are shown for the same case as Fig. 7. The bias is clearly correlated to the SRF position, and thus to the influence of 3-D radiative transfer effects. This scenario uses the soil surface, which has an albedo of 0.31 . The range in bias is about 0.03 , which is a relative bias of $10 \%$. The other retrieved surface albedos show similar relationships to the SRF position. Also note that the screening algorithms remove retrievals mostly around SRF 


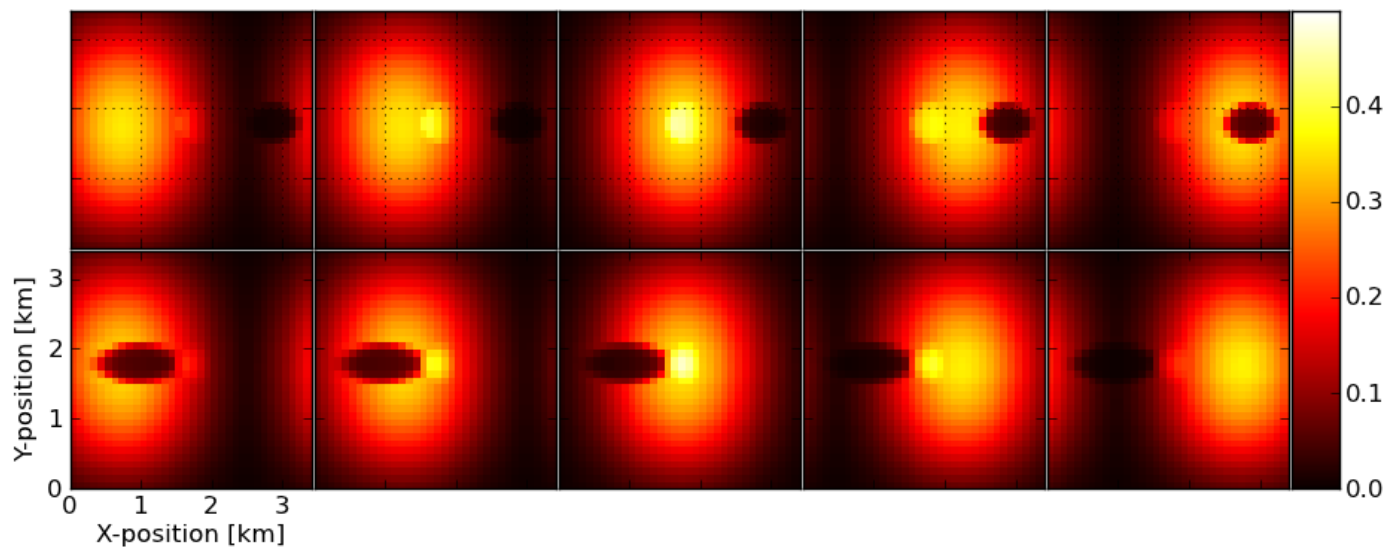

Figure 4. Overhead view of the scene reflectance multiplied by the SRF at positions $0,5,10,15$, and 20 , for the $0.6 \mathrm{~km}$ cloud in the $\mathrm{O}_{2}$ A-band over the soil surface. The SRF moves from left to right over the high spatial resolution grid. The top row shows the SRF sequence for the $35^{\circ}$ solar zenith angle (SZA), and the bottom row shows the sequence for the $60^{\circ} \mathrm{SZA}$. In the top row, the SRF starts at a position where the FOV is primarily filled with a "clear" surface view, to a "cloudy" position, and ends at a "shadowed" position. In the bottom row, the periodic boundary condition used in SHDOM causes the shadow to wrap around to the other side of the high spatial resolution scene for the larger SZA. For this case, the SRF starts in the "shadowed" position, followed by "cloudy" and then "clear".

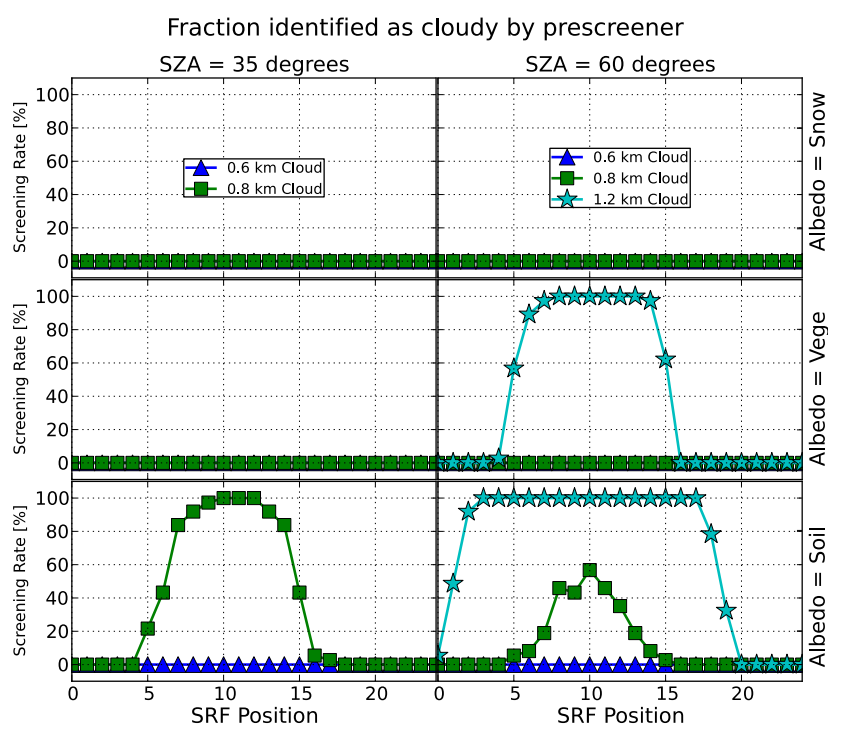

Figure 5. Fraction of simulated spectra identified as cloudy by the prescreening algorithm.

position 10 (the position centered on the cloud), which is also where the retrieved $\mathrm{SCO} 2$ albedo is the most accurate. The result is that the bias range, shown by the box plot, is not reduced.

The summary of SCO2 albedo biases is shown in Fig. 10. Although the albedo bias is within the range $0.01-0.02$ in all cases, the bias relative to the $\mathrm{SCO} 2$ albedo is substantial. A 0.01 albedo bias relative to the $0.02,0.10$, and 0.31 albedo values, for the snow, vegetated, and soil surfaces, respectively, is a relative bias of approximately 50,10 and $3 \%$.

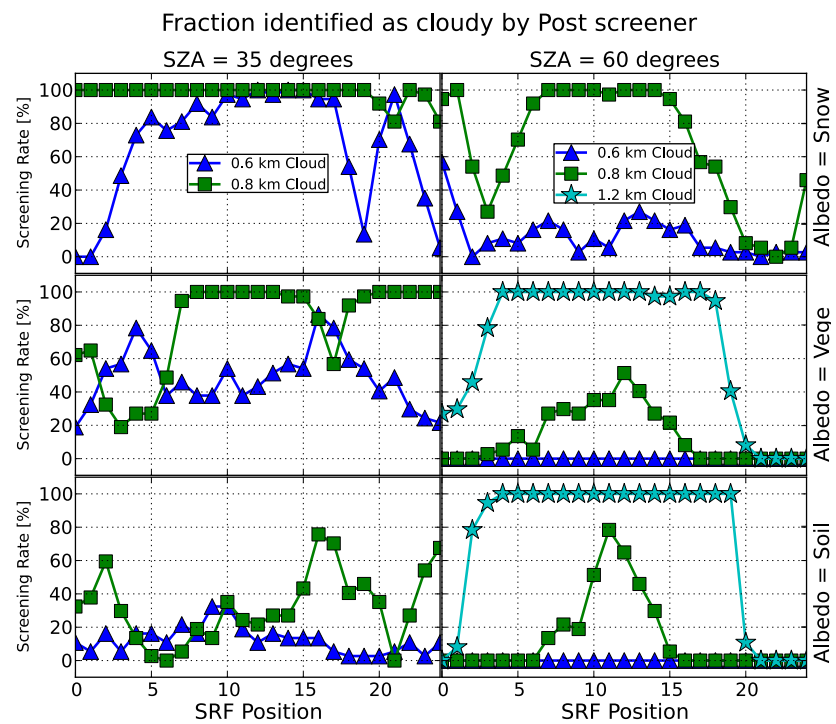

Figure 6. Fraction of simulated spectra identified as cloudy by the post screening algorithm.

\subsubsection{Surface pressure}

The final retrieved variable for $\mathrm{CO}_{2}$ concentration is the dry air mole fraction (the $X_{\mathrm{CO}_{2}}$ ), which is equivalent to the ratio of $\mathrm{CO}_{2}$ molecules and $\mathrm{O}_{2}$ molecules in the column. The latter value is represented by the surface pressure, since the $\mathrm{O}_{2}$ concentration varies much less than the $\mathrm{CO}_{2}$ concentration. This implies that any bias in the retrieved surface pressure will directly cause bias in the retrieved $X_{\mathrm{CO}_{2}}$. Plots for surface pressure bias are shown in Figs. 11 and 12, matching 


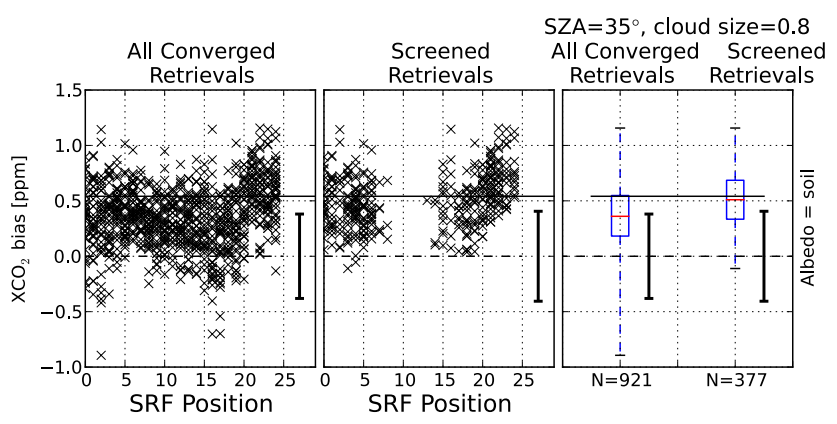

Figure 7. Bias in retrieved $X_{\mathrm{CO}_{2}}$, showing the scatter plot of all retrievals (left panel) and screened retrievals (middle panel), compared to the single box plot summarizing the bias (right panel). The error bar drawn with a thick black line, centered on zero bias, is the predicted posterior error $(1 \sigma)$ from the optimal estimation algorithm. The thin horizontal line marks the mean bias for retrievals from a cloud-free simulation. Finally, the number of retrievals in each box plot is indicated at the bottom of the $x$ axis in the right panel.

the bias plots in the previous sections for $X_{\mathrm{CO}_{2}}$ and $\mathrm{SCO} 2$ surface albedo.

Since surface pressure is used in the ABP and for postscreening, large surface pressure bias will cause the observation to be screened. In Fig. 11, the maximum absolute surface pressure bias is in the cloud-centered SRF positions. These are the most likely to be screened, so in this case the overall surface pressure bias is reduced somewhat by the screening algorithms. The summary plot, in Fig. 12, shows that the overall reduction in bias only occurs in a few scenarios (both SZA for cloud size $0.8 \mathrm{~km}$ over the soil surface). The general patterns in the surface pressure bias are different than those seen in $X_{\mathrm{CO}_{2}}$. For $X_{\mathrm{CO}_{2}}$, the soil surface retrievals show bias very similar to the clear sky bias, and then the bias increased as the $\mathrm{SCO} 2$ albedo decreased, resulting in very large bias over the snow surface. The results for surface pressure are more related to the $\mathrm{O} 2 \mathrm{~A}$ albedo, since most of the sensitivity to surface pressure comes from the $\mathrm{O} 2 \mathrm{~A}$ band measurement. Thus, the surface pressure bias for the retrievals over the snow surface is quite different than the vegetation and soil surface albedos. The absolute bias for the retrievals over the snow surface albedo is about $1.5 \mathrm{hPa}$ for the $35^{\circ} \mathrm{SZA}$ and less than $0.3 \mathrm{hPa}$ for the $60^{\circ} \mathrm{SZA}$. The bias for the retrievals over the vegetation and soil surfaces are similar, with nearly zero bias for the $0.6 \mathrm{~km}$ cloud and $35^{\circ} \mathrm{SZA}$, and larger absolute bias for the larger cloud size and SZA.

\subsection{4 $\quad X_{\mathrm{CO}_{2}}$ and surface pressure}

Since the $X_{\mathrm{CO}_{2}}$ is directly related to the surface pressure, the joint distribution of bias between these two variables is worth examining. Figure 13 shows scatter plots of the biases for these two variables. Each panel corresponds to a sin-
Table 4. Percentage of simulated observations identified as cloudy by postscreener algorithm.

\begin{tabular}{llcc}
\hline $\begin{array}{l}\text { Surface } \\
\text { Type }\end{array}$ & $\begin{array}{l}\text { Cloud } \\
\text { Size }\end{array}$ & $\begin{array}{c}\text { Postscreen Pct. } \\
\text { SZA }=35^{\circ}\end{array}$ & $\begin{array}{c}\text { Postscreen Pct. } \\
\text { SZA }=60^{\circ}\end{array}$ \\
\hline Snow & clear & 28.4 & 1.4 \\
Snow & $0.6 \mathrm{~km}$ & 67.0 & 12.5 \\
Snow & $0.8 \mathrm{~km}$ & 98.1 & 66.6 \\
Snow & $1.2 \mathrm{~km}$ & & 95.8 \\
\hline Vege & clear & 1.4 & 0.0 \\
Vege & $0.6 \mathrm{~km}$ & 48.2 & 0.0 \\
Vege & $0.8 \mathrm{~km}$ & 80.0 & 13.2 \\
Vege & $1.2 \mathrm{~km}$ & & 68.8 \\
\hline soil & clear & 2.7 & 0.0 \\
soil & $0.6 \mathrm{~km}$ & 12.5 & 0.0 \\
soil & $0.8 \mathrm{~km}$ & 32.3 & 13.2 \\
soil & $1.2 \mathrm{~km}$ & & 71.7 \\
\hline
\end{tabular}

gle scenario, matching the layout seen earlier in Fig. 8. The black points show the biases for the individual screened retrievals, and the large cyan cross shows the clear sky retrieval bias. The overplotted ellipse in the lower left corner is an example posterior covariance from the optimal estimation algorithm. The covariance is computed for each retrieval, but within each scenario the covariance does not change significantly between each retrieval. Note that the display scales in both the $x$ and $y$ axes are different in each row of plot panels (corresponding to a single surface albedo). The "flattened" appearance of the covariance ellipses for the snow surface albedo scenarios is largely due to the large $y$-range for the $X_{\mathrm{CO}_{2}}$ bias display. The ellipse orientation describes the degree of covariance between the two variables, as calculated by the optimal estimation algorithm. It is clear that there is no consistent strong covariance, but rather a weak covariance that is scenario dependent. The retrieval biases also show correlation that is dependent on the scenario. The correlation is highest in the $60^{\circ} \mathrm{SZA}$, vegetation scenario, and very low in all scenarios using the soil albedo.

\subsubsection{Profiles of atmospheric scatterers}

The optimal estimation retrieval has parameterized profiles of four atmospheric scatterers (see Sect. 3). Figure 14 shows the retrieved profiles of cloud water (top row), cloud ice (middle row) and aerosol (bottom row) optical depth. Since the two aerosol types were typically strongly correlated, the profiles were summed and the combined profile is shown in Fig. 14. The optical depths per layer are represented by the color scale, ranging from 0 to 0.003 . Vertical pressure levels are given on the $y$ axis, and the SRF positions are given on the $x$ axis. Each column represents the average retrieved optical depth profile for that SRF position and simulation scenario. The four cloud and SZA scenarios using the soil 

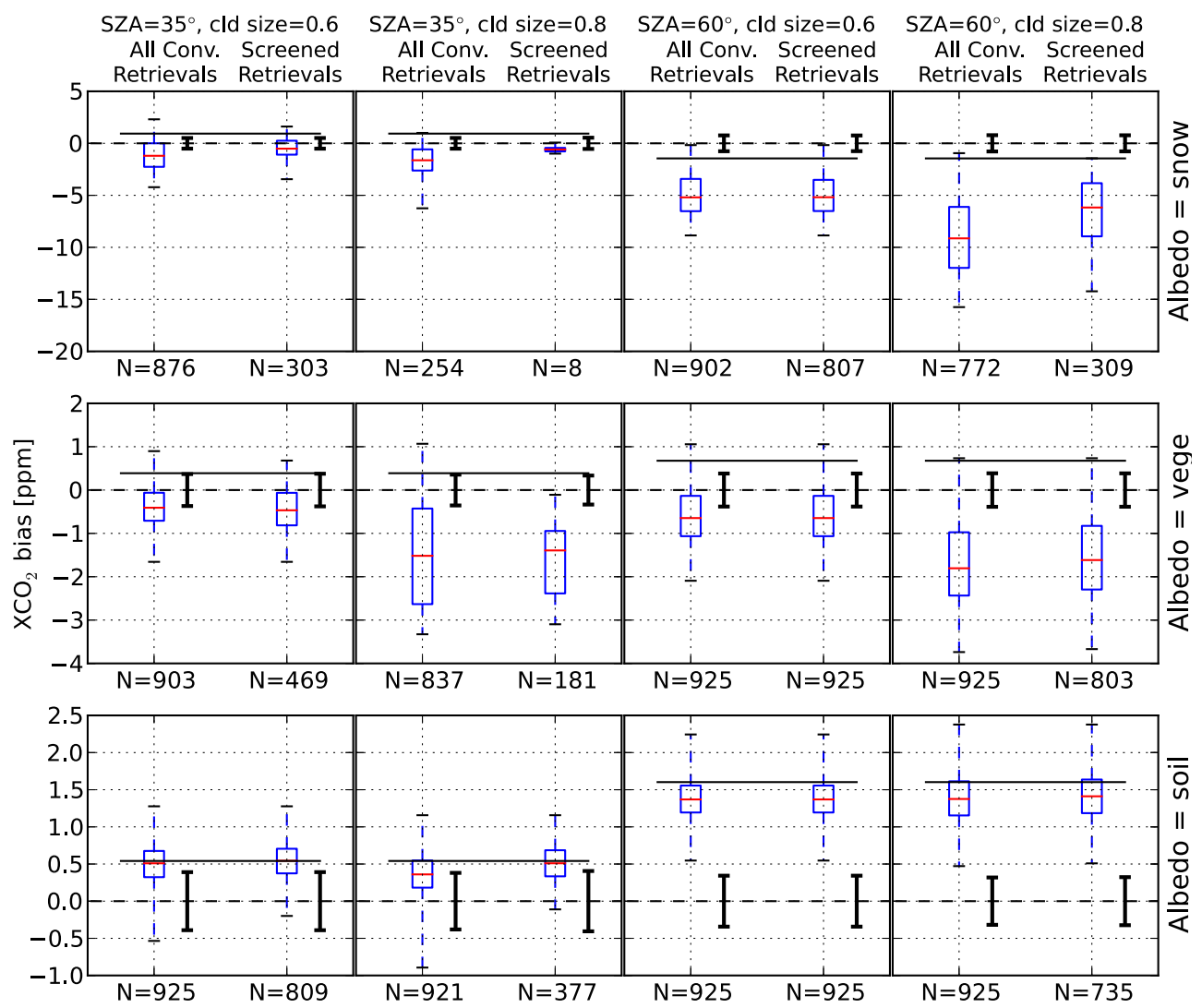

Figure 8. Summary of $X_{\mathrm{CO}_{2}}$ retrieval bias for all scenarios with 0.6 and $0.8 \mathrm{~km}$ clouds. See Fig. 7 for details.

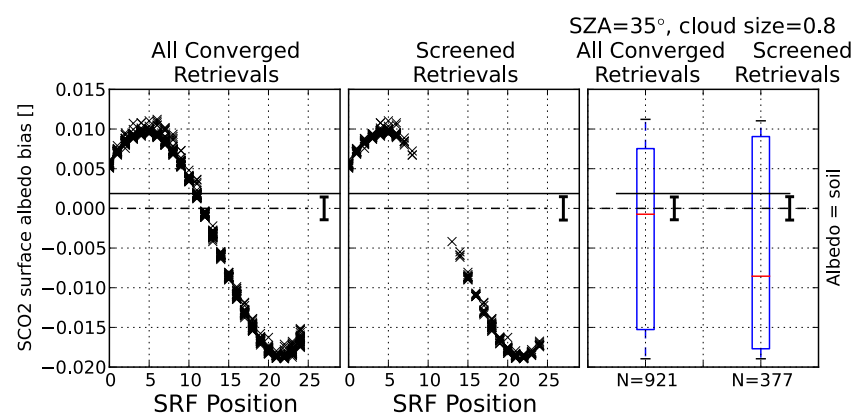

Figure 9. Bias in retrieved $\mathrm{SCO} 2$ albedo, with the same layout as Fig. 7.

surface are shown. Note that the retrieval estimates a significant amount of cloud ice optical depth at high altitudes, even though the simulation did not include any cloud ice at any level, and no scatterers above the $700 \mathrm{hPa}$ level other than molecular scattering. The gap in the middle of the second column (the $0.8 \mathrm{~km}$ cloud size, and $35^{\circ} \mathrm{SZA}$ ) is due to the complete screening of these retrievals.

\section{Discussion}

The overall purpose of the prescreening algorithm is to quickly identify observations that are sufficiently contaminated by cloud to preclude accurate Level $2 X_{\mathrm{CO}_{2}}$ retrievals. Characterizing its performance for the scenarios here must involve a discussion of the prescreening rate as well as the $X_{\mathrm{CO}_{2}}$ bias with and without applying the prescreening.

In general, the ABP cannot detect the smallest sub-FOV cloud $(0.6 \mathrm{~km}$ diameter $)$, and has limited ability to detect the larger clouds ( 0.8 and $1.2 \mathrm{~km}$ diameter). It is important to note however that the reasonable accuracy of the ABP algorithm in identifying homogeneous cloudy scenes, i.e, $100 \%$ FOV contamination, has been shown on simulated data (O'Dell et al., 2012). Furthermore, comparisons were favorable against the MODIS cloud mask for select GOSAT soundings (Taylor et al., 2012). The screening fraction is related to the albedo in the $\mathrm{O} 2 \mathrm{~A}$ band, as the screening rates are highest for the soil surface, and zero for the snow surface (see Table 3 and Fig. 5). Likely this is due to poor contrast between the surface and cloud when the surface is bright (for example, in Fig. 3 the cloud is barely discernible over the snow surface in the $\mathrm{O} 2 \mathrm{~A}$ band). For the snow surface, the $X_{\mathrm{CO}_{2}}$ biases are often quite large, up to 5-10 ppm, so the ABP is not effective within the scenarios over snow. Screen- 


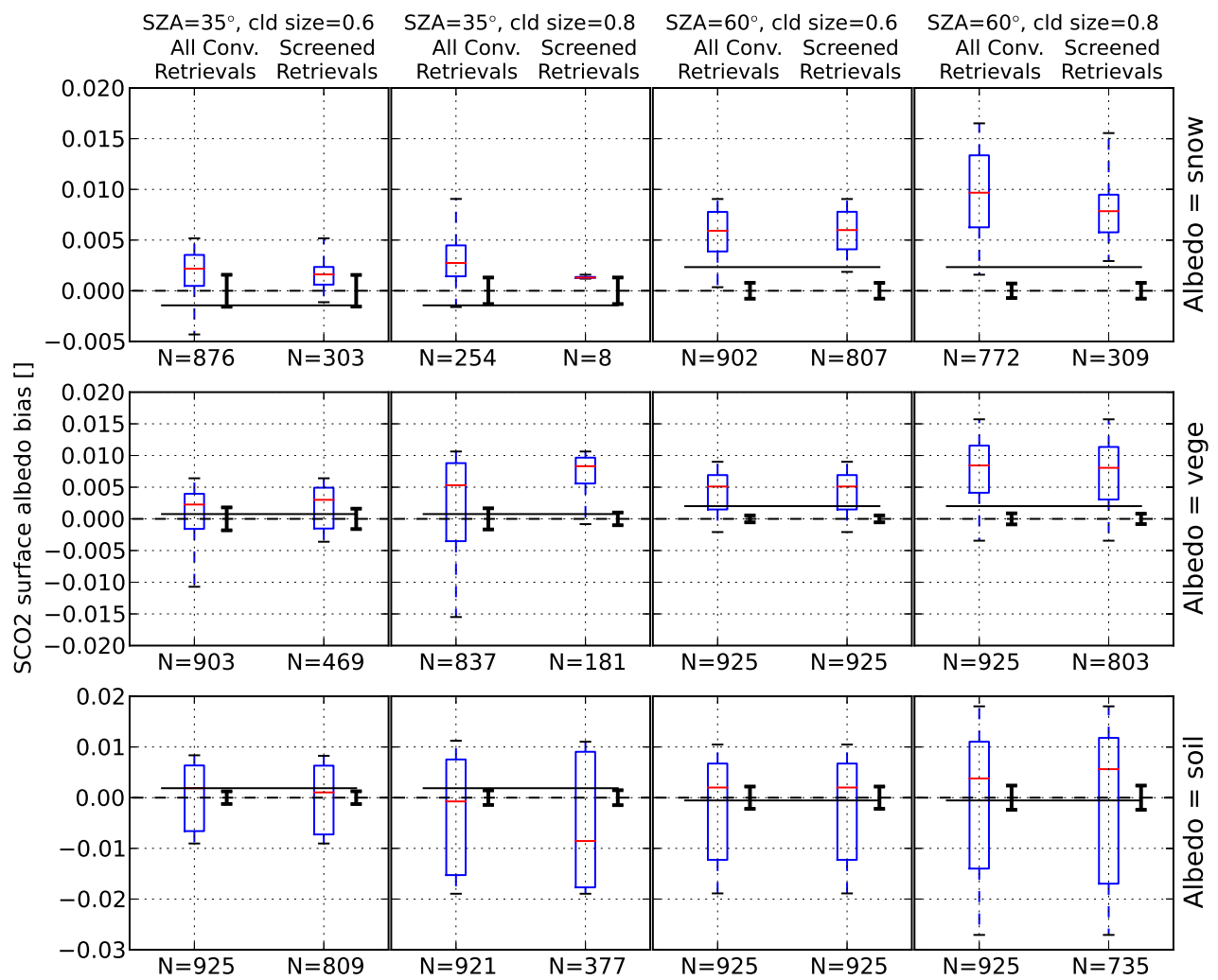

Figure 10. Summary of SCO2 surface albedo retrieval bias for all scenarios with 0.6 and $0.8 \mathrm{~km}$ clouds, with the same layout as Fig. 8 .

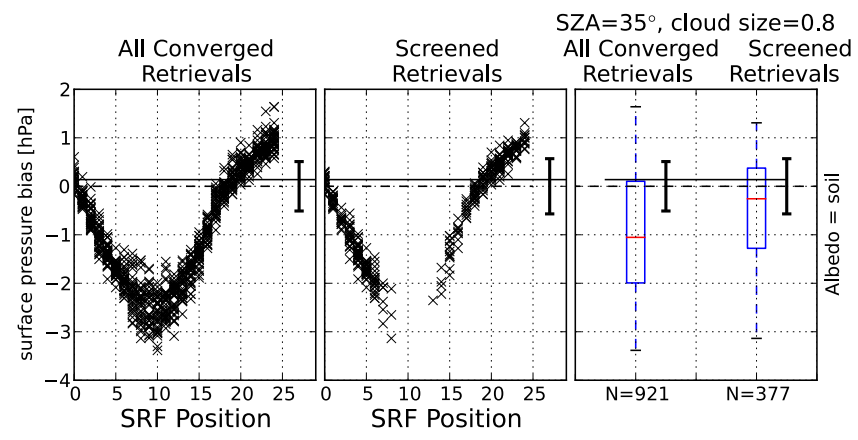

Figure 11. Bias in retrieved surface pressure, with the same layout as Fig. 7.

ing out retrievals over snow surfaces could easily be achieved by other simple tests. For example, filtering could be done seasonally by latitude to exclude retrievals over likely snow covered regions, or by requiring a minimum signal level in the SCO2 band. Operationally, the ACOS retrieval algorithm has taken similar steps to avoid retrievals over snow and ice surfaces, by using thresholds on the retrieved albedos to exclude likely snow or ice covered surfaces. These thresholds remove retrievals with very low $\mathrm{SCO} 2$ albedo, or retrievals with a combination of very high $\mathrm{O} 2 \mathrm{~A}$ albedo and low $\mathrm{SCO} 2$ albedo. Over the vegetated surface, the biases are smaller, but still significant, up to 2 ppm to the known truth value and up to $3 \mathrm{ppm}$ relative to the clear sky bias value. These scenarios are the most concerning for the ABP. The soil surface, in contrast, yields very accurate $X_{\mathrm{CO}_{2}}$ retrievals, with bias less than $1.5 \mathrm{ppm}$ relative to the known truth value and within $0.3 \mathrm{ppm}$ relative to the clear sky bias value. In fact, the L2 retrieval yields accurate $X_{\mathrm{CO}_{2}}$ retrievals even for the prescreened observations, indicating the ABP is too restrictive in some cases.

The postscreen filters applied after the L2 retrieval are much more effective at identifying the cloud contamination. In nearly all surface and SZA scenarios the $0.8 \mathrm{~km}$ cloud is identified in more than half of the observations. The vegetated and soil surfaces for $60^{\circ}$ solar zenith angle are the exception, but the majority of cases are screened at the $1.2 \mathrm{~km}$ cloud size.

The $X_{\mathrm{CO}_{2}}$ biases on the final screened retrievals are generally equivalent to the bias from the set of all converged retrievals. This is illustrated with the box plots in Fig. 8. If the screening methods were preferentially identifying high $X_{\mathrm{CO}_{2}}$ bias retrievals, then the box plot for the screened retrieval set should be narrower and closer to the clear sky bias. However, in all cases the two box plots are equivalent.

For other retrieved state variables, the bias is more significant. The SCO2 albedo bias results shown in Figs. 9 and 10 show similar behaviors as the $X_{\mathrm{CO}_{2}}$ bias. Specifically, the 

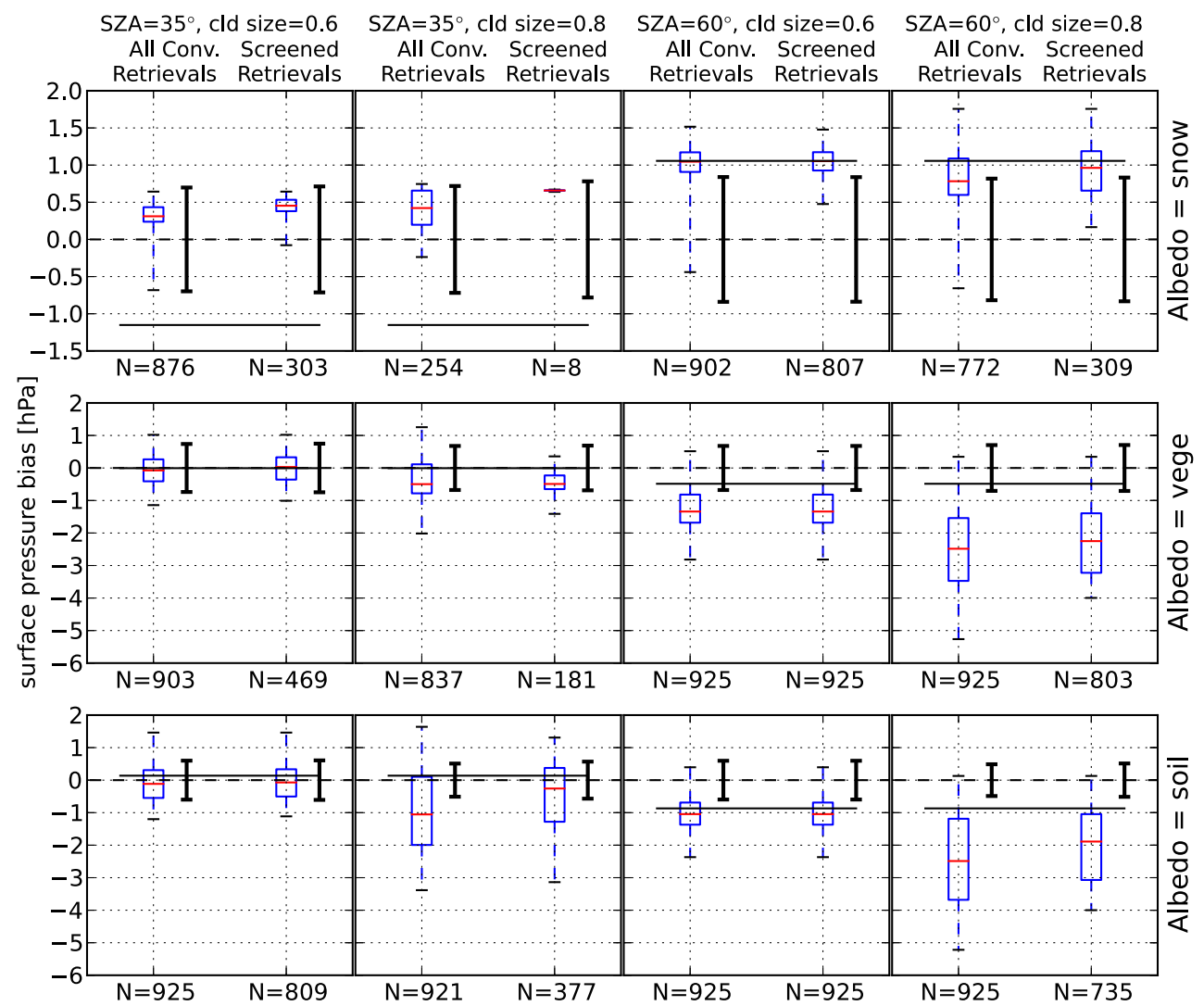

Figure 12. Summary of surface pressure retrieval bias for all scenarios with 0.6 and $0.8 \mathrm{~km}$ clouds, with the same layout as Fig. 8 .

bias is largest for snow, and smallest for soil, and the screening does not reduce the mean absolute bias. The latter effect can be clearly seen in Fig. 9, since the screening tends to remove observations at SRF position 10 (centered on the cloud), where the retrieved $\mathrm{SCO} 2$ albedo is close to the truth value.

The retrieved optical depth profiles for the scattering particles also exhibit large biases. Figure 14 shows the results for four soil surface cases $(0.6$ and $0.8 \mathrm{~km}$ clouds at both solar zenith angles). The profiles show complicated relationships to the SRF positions, with both the total optical depths and the profile altitudes changing as the SRF scans over the scene. Note that from the screening rate results (Tables 3 and 4) most of these retrievals are passed by both screening algorithms. Since the reduced $\chi^{2}$ was used as part of the postscreen algorithm, the spectral residuals are small. Thus the 1-D radiative transfer model is able to recreate the spectra simulated with the 3-D radiative transfer code well enough to produce low $\chi^{2}$ values and pass the screening algorithm. Thus the patterns in the optical depth profiles represent different 1-D plane parallel representations of the 3-D scattering field in the simulations. These scatterer profiles are very different from the true 3-D distributions. Recall from Sect. 4 that the aerosol layer in the model is evenly distributed in the lower $2.5 \mathrm{~km}$, which is the approximately the pressure layer for $P>700 \mathrm{hPa}$, while the cloud is located at an altitude of $1.6 \mathrm{~km}$ (approximately $800 \mathrm{hPa}$ ). The retrieved scatterer profiles are displaced vertically as the SRF position moves across the cloud, but the actual scatterers are at fixed altitudes. Only the results for the retrievals over the soil surface are shown here. The retrievals over other surfaces show qualitatively similar behavior, in that the scatterer vertical profiles change significantly with the SRF position.

\section{Conclusions}

By processing simulated OCO-2 observations created with a 3-D radiative transfer model, the impact of 3-D scattering effects has been estimated for the scenario of low altitude, sub-FOV liquid water clouds. Tests were done for two solar zenith angles $\left(35\right.$ and $\left.60^{\circ}\right)$, and three surface types (snow, vegetation, and soil). Overall, the retrieved $X_{\mathrm{CO}_{2}}$ shows biases that are strongly dependent on the $\mathrm{SCO} 2$ surface albedo. After screening, the worst case mean bias over snow is roughly $5 \mathrm{ppm}$ relative to clear sky retrievals, for the $0.8 \mathrm{~km}$ cloud and $60^{\circ}$ solar zenith angle. At the other extreme, the mean retrieved $X_{\mathrm{CO}_{2}}$ bias is less than $0.5 \mathrm{ppm}$ relative to the clear sky retrieval in all cases. The performance of the screening algorithms is highly scene dependent, and no clear general trend is evident in the data. The 

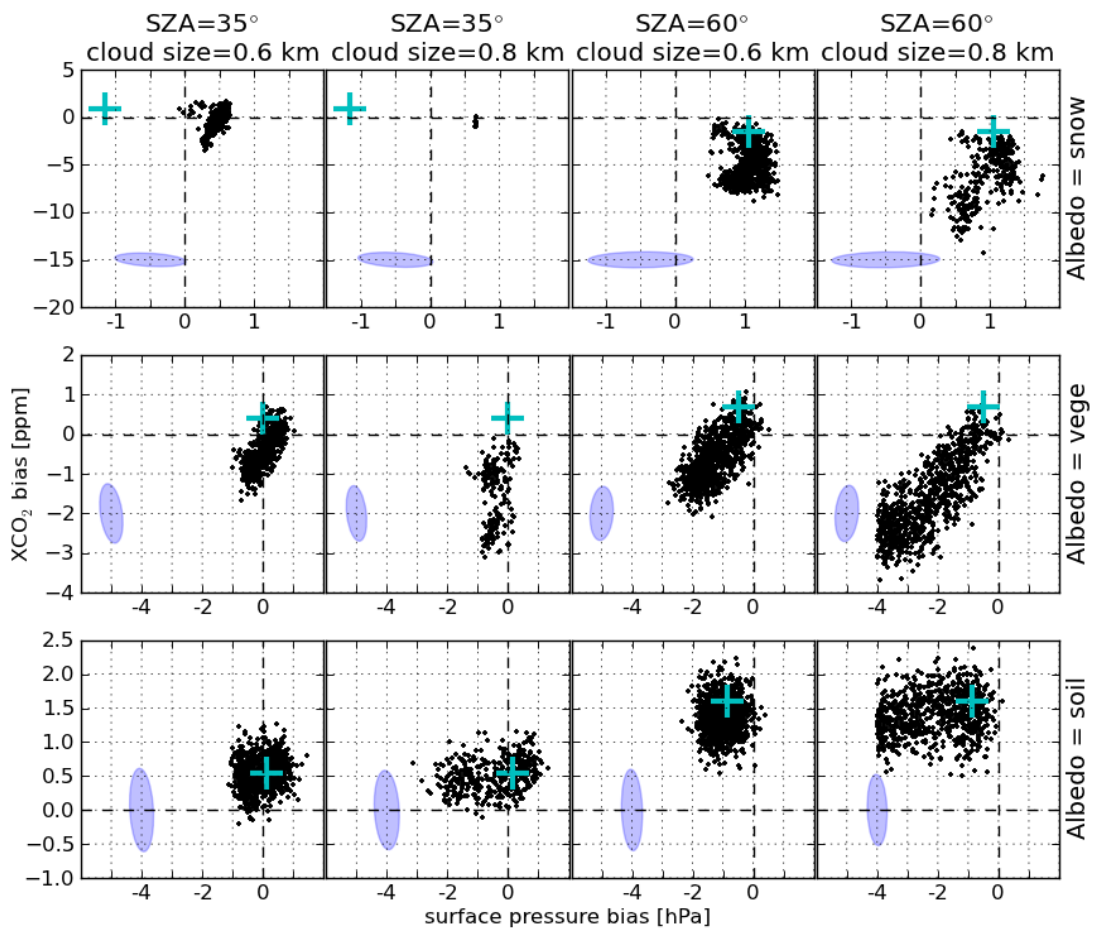

Figure 13. Summary of surface pressure and $X_{\mathrm{CO}_{2}}$ retrieval bias and covariance for all scenarios with 0.6 and $0.8 \mathrm{~km}$ clouds. Plot panels are arranged in the same order as Fig. 8. In each panel, the screened retrievals are shown (black points) along with the mean clear sky retrieval (large cyan cross). The ellipse shows the $1 \sigma$ posterior covariance for these two retrieved variables, offset by an arbitrary amount for display clarity.

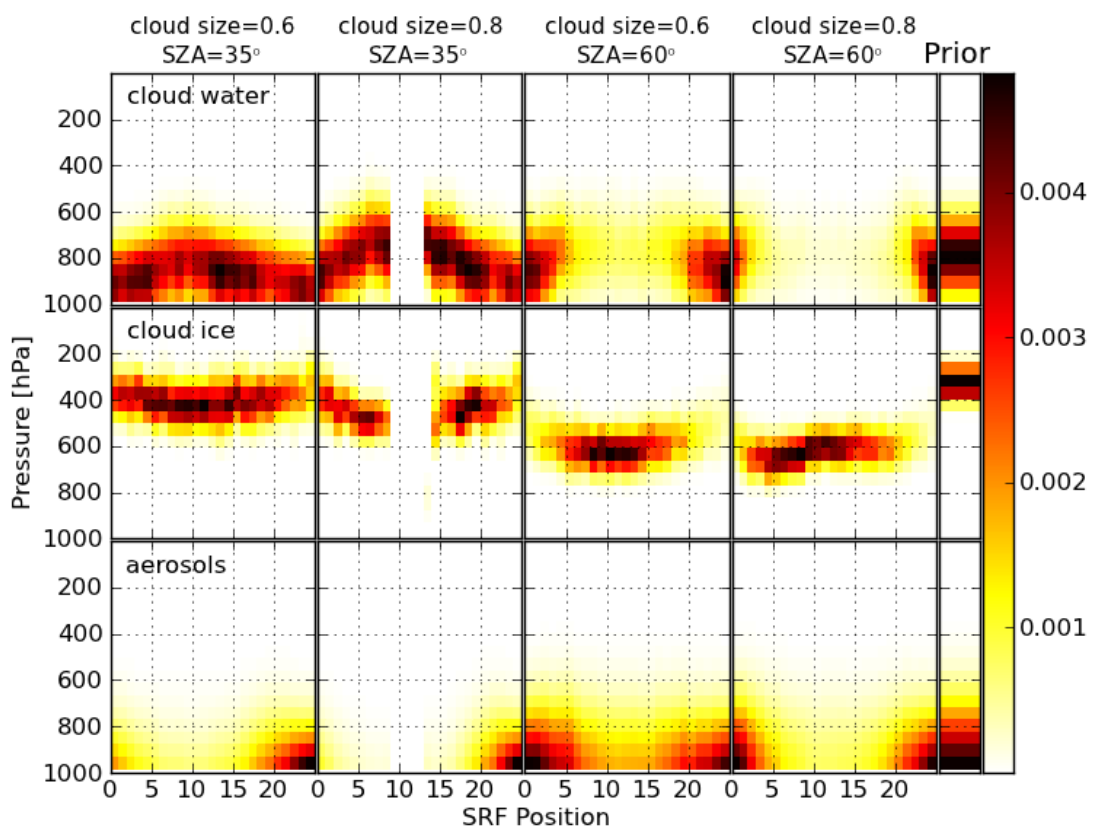

Figure 14. Retrieved optical depth profiles of atmospheric scatterers for four scenarios (cloud sizes 0.6 and $0.8 \mathrm{~km}$ for both SZA) with the soil surface albedo. Each panel shows the profile in a vertical column, with the $y$ axis indicating profile pressure, and the $x$ axis indicating the SRF position. Top, middle and bottom rows show the cloud water, cloud ice, and aerosol (sum of the Khan $2 \mathrm{~b}$ and $3 \mathrm{~b}$ ) profiles, respectively. The far right column shows the prior optical depth profiles used by the optimal estimation retrieval. 
ABP has difficulty identifying these sub-FOV clouds, but in many cases the L2 retrieval is still able to retrieve an accurate $X_{\mathrm{CO}_{2}}$. Neither screening method reduces the mean bias within a single test case. From the overall perspective of the entire group of tests, the screening methods do reduce bias by screening higher fractions of the larger clouds sizes which do have larger $X_{\mathrm{CO}_{2}}$ bias. Both screening methods primarily detect the cloud-centered SRF positions, so the shadowcontaminated observations are detected less often.

While the analysis presented here does indicate potentially large biases in the OCO-2 $X_{\mathrm{CO}_{2}}$ retrievals, at present we do not know the potential impact on the global $X_{\mathrm{CO}_{2}}$ data set. A future study is needed to connect these results to measured spatial and temporal distribution of clouds. The resulting bias in a global $X_{\mathrm{CO}_{2}}$ data set will depend on the occurrence of cloudy scenes similar to the presented synthetic scenarios. The biases could be especially problematic if they are regionally correlated.

During this study, development has continued on the operational retrieval algorithm past version B3.4. The treatment of aerosols is quite different in more recent versions. A geographically dependent aerosol climatology is used to select the pair of aerosols used for each retrieval from a set of six aerosol types. Clearly the retrieval biases among the albedo and aerosol profiles are highly correlated, so the quantitative behavior may be very different in the new algorithm. However the general behavior of the retrieved aerosol profile where it is highly dependent on the unresolved 3-D cloud field - should remain unchanged.

Finally, one important caveat on these results is the reliance on an unpolarized forward model. Since the actual OCO-2 measurement is polarized, these results should be repeated with the same framework using a fully polarized 3-D radiative transfer model. It is unknown how the retrieval will behave differently when working with the $(I-Q) / 2$ radiance instead of the unpolarized radiance. In addition, it would be important to extend these analyses to the glint mode observation. Polarized radiative transfer is even more important for simulating glint mode observation, since the glint mode relies on the strongly polarized specular reflection from the ocean surface. Since this project began, the SHDOM model has been improved and now can perform the polarized radiative transfer (Evans, 2014).

Acknowledgements. This research project was supported by the NASA OCO-2 Science Team, under contract NNX13AB97G. The authors thank the Space Science and Engineering Center Technical Computing group for providing cluster computing resources used for the simulations.

Edited by: H. Worden

\section{References}

Bennartz, R. and Preusker, R.: Representation of the photon pathlength distribution in a cloudy atmosphere using finite elements, J. Quant. Spectrosc. Ra., 98, 202-219, 2006.

Bovensmann, H., Buchwitz, M., Burrows, J. P., Reuter, M., Krings, T., Gerilowski, K., Schneising, O., Heymann, J., Tretner, A., and Erzinger, J.: A remote sensing technique for global monitoring of power plant $\mathrm{CO}_{2}$ emissions from space and related applications, Atmos. Meas. Tech., 3, 781-811, doi:10.5194/amt3-781-2010, 2010.

Buchwitz, M., Schneising, O., Burrows, J. P., Bovensmann, H., Reuter, M., and Notholt, J.: First direct observation of the atmospheric $\mathrm{CO}_{2}$ year-to-year increase from space, Atmos. Chem Phys., 7, 4249-4256, doi:10.5194/acp-7-4249-2007, 2007.

Crisp, D. and Johnson, C.: The Orbiting Carbon Observatory mission, Acta Astronaut., 56, 193-197, doi:10.1016/j.actaastro.2004.09.032, 2005.

Crisp, D., Atlas, R., Breon, F.-M., Brown, L., Burrows, J., Ciais, P., Connor, B., Doney, S., Fung, I., Jacob, D., Miller, C., O'Brien, D., Pawson, S., Randerson, J., Rayner, P., Salawitch, R., Sander, S., Sen, B., Stephens, G., Tans, P., Toon, G., Wennberg, P., Wofsy, S., Yung, Y., Kuang, Z., Chudasama, B., Sprague, G., Weiss, B., Pollock, R., Kenyon, D., and Schroll, S.: The Orbiting Carbon Observatory (OCO) mission, Adv. Space Res., 34, 700-709, 2004.

Crisp, D., Bösch, H., Brown, L., Castano, R., Christi, M., Connor, B., Frankenberg, C., McDuffie, J., Miller, C. E., Natraj, V., O’Dell, C., O’Brien, D., Polonsky, I., Oyafuso, F., Thompson, D., Toon, G., and Spurr, R.: OCO (Orbiting Carbon Observatory)2 Level 2 Full Physics Retrieval Algorithm Theoretical Basis, 2010.

Crisp, D., Fisher, B. M., O'Dell, C., Frankenberg, C., Basilio, R., Bösch, H., Brown, L. R., Castano, R., Connor, B., Deutscher, N. M., Eldering, A., Griffith, D., Gunson, M., Kuze, A., Mandrake, L., McDuffie, J., Messerschmidt, J., Miller, C. E., Morino, I., Natraj, V., Notholt, J., O’Brien, D. M., Oyafuso, F., Polonsky, I., Robinson, J., Salawitch, R., Sherlock, V., Smyth, M., Suto, H., Taylor, T. E., Thompson, D. R., Wennberg, P. O., Wunch, D., and Yung, Y. L.: The ACOS $\mathrm{CO}_{2}$ retrieval algorithm - Part II: Global $X_{\mathrm{CO}_{2}}$ data characterization, Atmos. Meas. Tech., 5, 687-707, doi:10.5194/amt-5-687-2012, 2012.

Dubovik, O., Holben, B., Eck, T. F., Smirnov, A., Kaufman, Y. J., King, M. D., Tanré, D., and Slutsker, I.: Variability of absorption and optical properties of key aerosol types observed in worldwide locations, J. Atmos. Sci., 59, 590-608, 2002.

Evans, K. F.: The spherical harmonics discrete ordinate method for three-dimensional atmospheric radiative transfer, J. Atmos. Sci. 55, 429-446, 1998.

Evans, K. F.: Polarized SHDOM, American Meteorological Society, 14th Conference on Atmospheric Radiation, Boston, MA, July 2014, available at: https://ams.confex.com/ams/ 14CLOUD14ATRAD/webprogram/Paper249476.html (last access: 20 November 2014), 2014.

Hansen, J. E. and Travis, L. D.: Light scattering in planetary atmospheres, Space Sci. Rev., 16, 527-610, doi:10.1007/BF00168069, 1974. 
Kahn, R., Banerjee, P., and McDonald, D.: Sensitivity of multiangle imaging to natural mixtures of aerosols over ocean, J. Geophys. Res.-Atmos., 106, 18219-18238, 2001.

Mandrake, L., Frankenberg, C., O’Dell, C. W., Osterman, G., Wennberg, P., and Wunch, D.: Semi-autonomous sounding selection for OCO-2, Atmos. Meas. Tech., 6, 2851-2864, doi:10.5194/amt-6-2851-2013, 2013.

Miller, C. E., Crisp, D., DeCola, P. L., Olsen, S. C., Randerson, J. T., Michalak, A. M., Alkhaled, A., Rayner, P., Jacob, D. J., Suntharalingam, P., Jones, D. B. A., Denning, A. S., Nicholls, M. E., Doney, S. C., Pawson, S., Boesch, H., Connor, B. J., Fung, I. Y., O’Brien, D., Salawitch, R. J., Sander, S. P., Sen, B., Tans, P., Toon, G. C., Wennberg, P. O., Wofsy, S. C., Yung, Y. L., and Law, R. M.: Precision requirements for spacebased $X_{\mathrm{CO}_{2}}$ data, J. Geophys. Res.-Atmos., 112, D10314, doi:10.1029/2006JD007659, 2007.

Miller, S. D. and Stephens, G. L.: CloudSat instrument requirements as determined from ECMWF forecasts of global cloudiness, J. Geophys. Res.-Atmos., 106, 17713-17733, 2001.

O’Brien, D. M., Polonsky, I., O'Dell, C., and Carheden, A.: Orbiting Carbon Observatory (OCO), algorithm theoretical basis document: the OCO simulator, Technical report ISSN 0737-535285, Cooperative Institute for Research in the Atmosphere, Colorado State University, 2009.

O’Dell, C., Day, J., Pollock, R., Bruegge, C., O’Brien, D., Castano, R., Tkatcheva, I., Miller, C., and Crisp, D.: Preflight radiometric calibration of the orbiting carbon observatory, IEEE T. Geosci. Remote, 49, 2438-2447, 2011.

O’Dell, C. W., Connor, B., Bösch, H., O’Brien, D., Frankenberg, C., Castano, R., Christi, M., Eldering, D., Fisher, B., Gunson, M., McDuffie, J., Miller, C. E., Natraj, V., Oyafuso, F., Polonsky, I., Smyth, M., Taylor, T., Toon, G. C., Wennberg, P. O., and Wunch, D.: The ACOS $\mathrm{CO}_{2}$ retrieval algorithm - Part 1: Description and validation against synthetic observations, Atmos. Meas. Tech., 5, 99-121, doi:10.5194/amt-5-99-2012, 2012.

Taylor, T., O’Dell, C., O’Brien, D., Kikuchi, N., Yokota, T., Nakajima, T., Ishida, H., Crisp, D., and Nakajima, T.: Comparison of cloud-screening methods applied to GOSAT near-infrared spectra, IEEE T. Geosci. Remote, 50, 295-309, 2012.
Thompson, D. R., Chris Benner, D., Brown, L. R., Crisp, D., Malathy Devi, V., Jiang, Y., Natraj, V., Oyafuso, F., Sung, K., Wunch, D., Castaño, R., and Miller, C. E.: Atmospheric validation of high accuracy $\mathrm{CO}_{2}$ absorption coefficients for the OCO-2 mission, J. Quant. Spectrosc. Ra., 113, 2265-2276, 2012.

Tran, H. and Hartmann, J.-M.: An improved $\mathrm{O}_{2}$ A band absorption model and its consequences for retrievals of photon paths and surface pressures, J. Geophys. Res.-Atmos., 113, D18104, doi:10.1029/2008JD010011, 2008.

Várnai, T., Marshak, A., and Yang, W.: Multi-satellite aerosol observations in the vicinity of clouds, Atmos. Chem. Phys., 13, 38993908, doi:10.5194/acp-13-3899-2013, 2013.

Wunch, D., Toon, G. C., Blavier, J.-F. L., Washenfelder, R. A., Notholt, J., Connor, B. J., Griffith, D. W. T., Sherlock, V., and Wennberg, P. O.: The total carbon column observing network, Philos. T. R. Soc. A, 369, 2087-2112, 2011a.

Wunch, D., Wennberg, P. O., Toon, G. C., Connor, B. J., Fisher, B., Osterman, G. B., Frankenberg, C., Mandrake, L., O’Dell, C., Ahonen, P., Biraud, S. C., Castano, R., Cressie, N., Crisp, D., Deutscher, N. M., Eldering, A., Fisher, M. L., Griffith, D. W. T., Gunson, M., Heikkinen, P., Keppel-Aleks, G., Kyrö, E., Lindenmaier, R., Macatangay, R., Mendonca, J., Messerschmidt, J., Miller, C. E., Morino, I., Notholt, J., Oyafuso, F. A., Rettinger, M., Robinson, J., Roehl, C. M., Salawitch, R. J., Sherlock, V., Strong, K., Sussmann, R., Tanaka, T., Thompson, D. R., Uchino, O., Warneke, T., and Wofsy, S. C.: A method for evaluating bias in global measurements of $\mathrm{CO}_{2}$ total columns from space, Atmos. Chem. Phys., 11, 12317-12337, doi:10.5194/acp11-12317-2011, 2011b.

Yoshida, Y., Ota, Y., Eguchi, N., Kikuchi, N., Nobuta, K., Tran, H., Morino, I., and Yokota, T.: Retrieval algorithm for $\mathrm{CO}_{2}$ and $\mathrm{CH}_{4}$ column abundances from short-wavelength infrared spectral observations by the Greenhouse gases observing satellite, Atmos. Meas. Tech., 4, 717-734, doi:10.5194/amt-4-717-2011, 2011.

Zhang, Z., Ackerman, A. S., Feingold, G., Platnick, S., Pincus, R., and Xue, H.: Effects of cloud horizontal inhomogeneity and drizzle on remote sensing of cloud droplet effective radius: case studies based on large-eddy simulations, J. Geophys. Res.-Atmos., 117, D19208, doi:10.1029/2012JD017655, 2012. 